\title{
Internationale Best Practices als Vorbild für Deutschland
}

\subsection{Identifikation und Einordnung internationaler Best Practices}

Best Practices bezeichnen im Allgemeinen erfolgreiche, praxiserprobte Vorgehensweisen, die für eine Nachahmung geeignet sind. Zunächst wurden entlang der sieben Dimensionen Strategie, Organisation, Recht, Technologie, Transparenz, Kollaboration und Partizipation 48 Best Practices recherchiert. Die Ergebnisse sind der Übersichtlichkeit halber in Tabelle A.11 im Anhang dargestellt: Im Ergebnis zeigt sich, dass etwa $70 \%$ der Best Practices eine bis maximal zwei Kategorien abdecken. Von allen Best Practices sind allein $60 \%$ den Kategorien Technologie und Transparenz zuzuordnen. Dies zeigt deutlich, dass aktuell die meisten Ansätze nur einen bestimmten Bedarf adressieren: Nur $30 \%$ weisen eine gesamtheitlichere Abdeckung von drei oder mehr Kategorien auf. Die umfänglichsten Best Practices waren jene einzelner Länder, hier von Frankreich, Mexiko, Portugal, Slowakei, UK und Ukraine. Sie decken in der Regel fünf Kategorien ab. Da eine Analyse und Darstellung aller Länder den Rahmen der Arbeit sprengen würde, wurde sich auf die Länder fokussiert, die der EU angehören, eine parlamentarische Republik darstellen und eine ähnliche Staatsstruktur wie Deutschland aufweisen (föderal oder regionalisiert). So ist die Vergleichbarkeit am Ehesten gegeben. Damit verblieben Frankreich und die Slowakei für eine Detailanalyse. 


\subsection{Ausgewählte Länderbeispiele in Europa}

\subsubsection{Slowakei}

\subsubsection{Korruption in der Slowakei}

Die Slowakei wurde lange mit einer im Vergleich zu anderen EU-Staaten hohen Korruptionsquote in Verbindung gebracht (European Commission, 2016d, S. 192). Ein Beispiel hierfür ist der "Notice Board Scandal“ aus dem Jahr 2007: Das slowakische Innenministerium hatte eine Ausschreibung über etwa 120 Millionen Euro mit einem Aushang am Schwarzem Brett innerhalb des Ministeriums publik gemacht. So konnten nur Unternehmen mit Verbindungen zum Innenministerium an der Ausschreibung teilnehmen und letztlich gewann ein Unternehmen mit nachweislichen Verbindungen zum damaligen Parteichef der Regierungspartei Ján Slota (Clare et al., 2016, S. 5). Dieser und ähnliche Skandale führten zu Bürgerprotesten (Š́́poš, 2015). In deren Gefolge wiederum kam es zu weitreichenden Reformen, die den Bürgern mehr Einsichtsrechte ermöglichten beziehungsweise die Zentralisierung des Einkaufs fokussierten (European Commission, 2016d, S. 193). Im CPI-Ranking stieg die Slowakei um elf Plätze von Platz 66 im Jahr 2011 auf Platz 57 im Jahr 2018 (Transparency International e. V., 2011; 2018b); das Land verzeichnete damit einen der stärksten Aufwärtstrends weltweit.

Dies bedeutete allerdings nicht, dass es nach 2011 keine Korruption mehr gegeben hätte. Eine wahrgenommene Korruption bestätigten $66 \%$ der befragten Unternehmen gemäß Flash Eurobarometer aus 2014 für die nationale Ebene und $70 \%$ für die regionale/lokale Ebene (European Commission, 2014, S. 74 f.). Dies entspricht einer hohen empfundenen Korruption. Zum Teil hat das mit der politischen Struktur zu tun: Die Slowakei ist ein „regionalisierter Staat“, was bedeutet, dass kein vollständiger Föderalismus im Rahmen eines ZweiKammern-Systems vorliegt, es aber eine gewisse Autonomie im definierten Umfang gibt (Berge \& Grasse, 2004, S. 73). ${ }^{1}$ Die etwa 2700 Gemeinden sind in ihrer Beschaffung autonom, was die lokalen Korruptionsrisiken erhöht. Etwa 1000 der Gemeinden haben nur maximal 1000 Einwohner.

\footnotetext{
${ }^{1}$ Das durchgeführte Interview bestätigte ebenfalls, dass eine starke Dezentralisierung vorliegt (persönliches Experteninterview mit Isabelle Adam am 07. März 2019; Isabelle Adam ist als Analyst am Global Transparency Institute (GTI) in Ungarn tätig. Dort befasst sie sich hauptsächlich mit Anti-Korruption im öffentlichen Einkauf sowie der Auswirkung von Transparenzreformen).
} 
Der Anteil der Ausschreibungen am BIP von insgesamt 73,6 Milliarden Euro im Jahr 2013 betrug $12 \%$. Somit entfielen auf den öffentlichen Einkauf etwa 8,48 Milliarden Euro. $57 \%$ der Ausschreibungen liefen über die TED und betrafen folglich den Oberschwellenbereich, entsprechend entfielen $43 \%$ auf den nationalen Unterschwellenbereich (European Commission, 2016d, S. 189).

\subsubsection{Der rechtliche Weg der Slowakei zu mehr Transparenz im öffentlichen Einkauf}

Die Slowakei war im Jahr 2006 eines der ersten zentraleuropäischen Länder mit einem eigenen Vergabegesetz (Dekret 25/2006) nach den Vorgaben der damaligen EU-Richtlinien (European Commission, 2016d, S. 189). Die Slowakei war folglich sehr schnell in der Umsetzung der europäischen Vorgaben in nationales Recht. Schon Jahre zuvor hatte die Einführung des Informationsfreiheitsgesetzes (Dekret 211/2000) im Jahr 2000 erstmalig für eine Eindämmung der Korruption sorgen sollen. Unter Berufung auf dieses Gesetz konnte jede Person oder Organisation staatliche Informationen anfordern, allerdings entsprach dies noch dem Ansatz „Transparenz auf Nachfrage“. Im Jahr 2011 wurde mit der Einführung des Dekrets 546/2010 ein Wandel hin zur proaktiven Veröffentlichung von Informationen angestoßen. Das Dekret erklärt die Online-Veröffentlichung des Großteils aller öffentlichen Verträge als verpflichtend - die Regelung geht sogar so weit, dass solche Verträge gar nicht in Kraft treten beziehungsweise ungültig werden, sofern sie nicht veröffentlicht sind (Nationalrat der Slowakischen Republik, 2010; Schneider, 2015, S. 3 f.; Šípoš et al., 2015). ${ }^{2}$

Ebenfalls 2011 trat die Slowakei der OGP bei, bekannte sich zu hundertprozentiger Offenheit im öffentlichen Einkauf und untermauerte dies mit entsprechenden Aktionsplänen rund um die Öffnung von Regierungsdaten (Office of the Plenipotentiary of the Government of the Slovak Republic for the Development of the Civil Society, 2017; Open Contracting Partnership, 2019h; Schneider, 2015). Hierzu gehörte auch die Erstellung des eigenen Open Data-Portals https:// data.gov.sk/ (Národná agentúra pre siet’ové a elektronické služby, 2019a). Dies umfasste im Oktober 2019 etwa 2100 offene Datensätze unterschiedlicher Qualität (Národná agentúra pre siet’ové a elektronické služby, 2019b). Im Experteninterview $^{3}$ stellte sich heraus, dass häufig Daten fehlen, zum Beispiel die Anzahl aller Mitbewerber, das Vertragsunterzeichnungsdatum und Daten von vor 2011.

\footnotetext{
${ }^{2} \S 47$ a Abs. 1 und 4 des Dekrets 546/2010.

${ }^{3}$ Persönliches Experteninterview mit Isabelle Adam (GTI Ungarn) am 07. März 2019.
} 
Um die Datenqualität zu erhöhen, wurden Gegenmaßnahmen im Nationalen Aktionsplan 2017-2019 beschlossen, zum Beispiel die Qualifizierung der Datenbereitsteller (Office of the Plenipotentiary of the Government of the Slovak Republic for the Development of the Civil Society, 2017, S. 9).

Darüber hinaus gibt es seit 2013 ein Datenschutzgesetz (Dekret 122/2013). Dieses sieht ebenfalls vor, dass Verträge veröffentlicht werden müssen, sobald eine der im Gesetz benannten Organisationen hierzu verpflichtet ist. Dies sind alle öffentlichen Einrichtungen auf allen Staatsebenen, Staatsunternehmen und Unternehmen, die ursprünglich von der öffentlichen Hand gegründet, aber später privatisiert wurden (Nationalrat der Slowakischen Republik, 2013). Des Weiteren muss der Vertrag mit öffentlichen Finanzierungen zusammenhängen (Allen \& Overy LLP, 2011). Laut Interview gab es nach der Offenlegung viel Protest und die Grauzone der Betriebsgeheimnisse sei teilweise von staatseigenen Firmen ausgenutzt worden, die für sich Ausnahmen geltend machen wollten. ${ }^{4}$

\subsubsection{Die Öffnung des öffentlichen Einkaufs in der Slowakei}

Der öffentliche Einkauf ist im Zuge der oben genannten Reformen stark zentralisiert worden (European Commission, 2016d). So verfügt die Slowakei über ein zentralisiertes Vergabesystem und eine zentrale Beschaffungsbehörde, das „Office for Public Procurement“, mit über 200 Beamten. Die Aufgaben der Behörde reichen von der Kontrolle der Rechtmäßigkeit der Vergabeverfahren über die jährliche Veröffentlichung von Vergabestatistiken bis hin zur Verhängung von Finanzsanktionen bei Verstößen (Office for Public Procurement, 2019). Der Bereich oberhalb der Wertgrenzen wird in Tabelle 9.1 angegeben (OTC; ,over the thresholds contracts"). In der Slowakei wird dabei nach dem Staat Slowakei als Einkäufer unterschieden, nach dem öffentlichen Einkauf (,Public procurer“), dem Einkäufer („Procurer") und allen Einkäufern (,all procurers“)

\footnotetext{
${ }^{4}$ Persönliches Experteninterview mit Isabelle Adam (GTI Ungarn) am 07. März 2019.

${ }^{5}$ Ein „öffentlicher Einkäufer“ umfasst den Staat Slowakei, die Regionen, Kommunen, öffentliche Kooperationen untereinander sowie Unternehmen im öffentlichen Interesse ohne Gewinnerzielungsabsicht. Ein „Einkäufer“ ist ein privates Unternehmen, welches durch den öffentlichen Einkauf finanziert und kontrolliert wird oder einen Sonderstatus per Gesetz besitzt und ein Geschäft innerhalb von sieben definierten Sektoren (zum Beispiel Transport, Post, Energie) betreibt (Miskuf et al., 2017, S. 16).
} 
Tabelle 9.1 Schwellenwerte, Einkäufer und Vertragsdefinition (in Anlehnung an Miskuf et al., 2017, S. 17, übersetzt durch Verfasserin)

\begin{tabular}{|c|c|c|}
\hline Wertgrenze & Einkäufer & Vertragsdefinition \\
\hline 135.000 Euro & Slowakei & Lieferung von Gütern und Dienstleistungen \\
\hline 135.000 Euro & Öffentlicher Einkäufer & $\begin{array}{l}\text { Lieferung von gesetzlich definierten Gütern } \\
\text { der Verteidigung }\end{array}$ \\
\hline 209.000 Euro & $\begin{array}{l}\text { Öffentlicher Einkäufer } \\
\text { (außer Slowakei) }\end{array}$ & Lieferungen von Gütern und Dienstleistungen \\
\hline 209.000 Euro & Öffentlicher Einkäufer & $\begin{array}{l}\text { Lieferungen von gesetzlich nicht definierten } \\
\text { Gütern der Verteidigung }\end{array}$ \\
\hline 750.000 Euro & Öffentlicher Einkäufer & $\begin{array}{l}\text { Lieferungen von gesetzlich definierten } \\
\text { Dienstleistungen }\end{array}$ \\
\hline 418.000 Euro & Einkäufer & Lieferungen von Gütern und Dienstleistungen \\
\hline 1.000.000 Euro & Einkäufer & $\begin{array}{l}\text { Lieferungen von gesetzlich definierten } \\
\text { Leistungen der Verteidigung }\end{array}$ \\
\hline 5.225.000 Euro & Alle Einkäufer & Bauleistungen \\
\hline 5.225.000 Euro & Alle Einkäufer & Konzessionen \\
\hline 135.000 Euro & Slowakei & Blueprint Wettbewerb \\
\hline 209.000 Euro & $\begin{array}{l}\text { Öffentlicher Einkäufer } \\
\text { (außer Slowakei) }\end{array}$ & Blueprint Wettbewerb \\
\hline 418.000 Euro & Einkäufer & Blueprint Wettbewerb \\
\hline
\end{tabular}

Die nationalen Wertgrenzen (Unterschwelle) sind in Tabelle 9.2 aufgeführt. Oberhalb dieser Grenzen und unterhalb jenen in Tabelle 9.1 handelt es sich um den nationalen Unterschwellenbereich UTC (,under the thresholds contracts“). Unterhalb dieser Wertgrenzen sind die Kleinstverträge LVC (,low value contracts“) angesiedelt (Miskuf et al., 2017, S. 18).

Tabelle 9.2 Wertgrenzen und Vertragsdefinitionen UTC (in Anlehnung an Miskuf et al., 2017, S. 18, übersetzt durch Verfasserin)

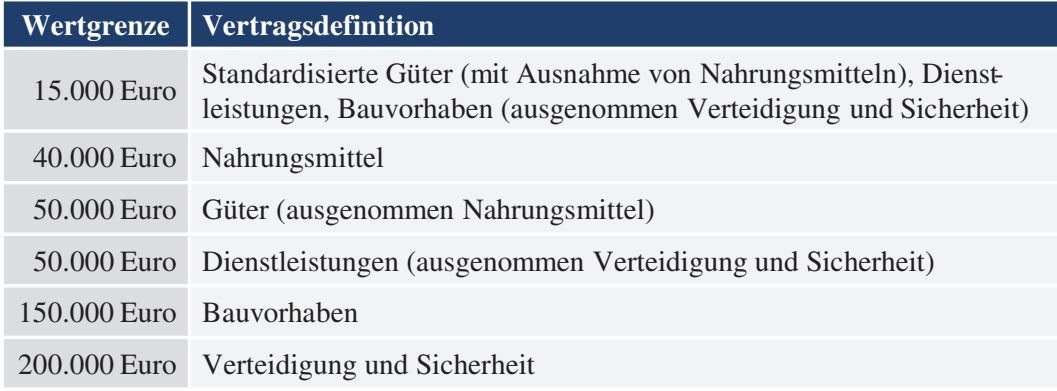


Die Ausschreibungen für standardisierte Güter und Dienstleistungen für den Unterschwellenbereich müssen über das ECS (Electronic Contracting System; https://www.eks.sk/Stranka/Information_of_EKS) als Online-Portal abgewickelt werden (Ausnahme: Nahrungsmittel), für den Oberschwellenbereich und Kleinstvergaben (LVC) ist dies nicht obligatorisch (XEKS, 2019). ECS ist eine zentrale Handelsplattform, die verpflichtend für den öffentlichen Einkauf ist. Sie bietet die voll automatisierte Auftragsabwicklung und besteht aus den SubSystemen eines elektronischen Marktplatzes, eines dynamischen Einkaufssystems sowie elektronischer Unterstützung in der Informationsbereitstellung und Kommunikation. ECS ist wiederum ein Teil von EVO (http://www.uvo. gov.sk/portal-evo), welches das zentrale E-Procurement-Portal der Slowakei ist. Die E-Procurement-Aktivitäten der Slowakei sind insgesamt in eine zentrale E-Government-Strategie eingebettet (European Commission, 2016a).

Die Prozeduren für den Unterschwellenbereich sind sehr viel weniger formalisiert. Laut einer Erhebung aus 2012 sind folgende Informationen zu veröffentlichen (Budapest Institute for Policy Analysis Ltd., 2012, S. 24):

- Ausschreibungsunterlagen

- Vergabeverfahren

- Leistungsbeschreibung

- Präqualifizierungsdokumente

- Präqualifizierungsbewertungen

- Angebots- und Teilnahmefrist

- Kriterien zur Bewertung der Qualität

- Vertragsvolumen

- Zuschlagskriterien

- Finaler Bewertungsreport

- Angabe der Komiteemitglieder, welche die Angebote bewertet haben

- Anzahl vergebener Verträge

- Unterschriebener Vertrag und weitere Modifikationen

- Vertragsnummer

- Name, Vorname des Gewinners/Verlierers; Identitätsnummer des Lieferanten, alternativ Geburtsdatum des Lieferanten

- Kopien der Angebotsdokumente

- Anzahl vergebener Verträge

- Keine Unterlagen hinsichtlich Leistung oder Vertragserfüllung

- Keine Unterlagen bezüglich Klagen gegen das Vergabeverfahren oder Zuschlagsempfehlungen 
Die zu veröffentlichenden Informationen betreffen demnach die Prozessstufen Ausschreibung, Bewertung und Vergabe, jedoch nicht die Planung und Ausführung.

Der Transparenz dienlich ist, dass die Bereitstellung der Daten eher anhand von Text- und weniger anhand von PDF-Formaten erfolgt: „Slovakia is the only country where the electronic bulletin is published in a way to create an appropriate and accurate, machine-readable, automatic database on public procurement" (Budapest Institute for Policy Analysis Ltd., 2012, S. 23). Dies unterstützt die datengetriebene Auswertung zur Kontrolle durch zum Beispiel NGOs und hilft dabei, selbst entwickelte Apps mit Daten zu bestücken (zum Beispiel https://tender.sme.sk/en/) (Transparency International e. V., 2019c) ${ }^{6}$. Des Weiteren ist es verpflichtend, die Verträge in einem zentralen Vertragsregister (https://www.crz.gov.sk/) (Government Office of the Slovak Republic, 2019) zu hinterlegen.

Flankiert wurden diese Bemühungen durch Maßnahmen in der Partizipation Dritter. Im Jahr 2015 wurde die Web-Anwendung Electronic Collective Petition (ECP) auf dem OGD-Portal http://www.slovensko.sk gestartet. Hierüber können Bürger ihre Anforderungen eingeben. Die Regierung muss diese berücksichtigen, wenn mindestens 15000 Unterstützer in 30 Tagen erreicht wurden. Sofern die Anforderungen die Exekutive betreffen, muss innerhalb von 60 Tagen eine Rückmeldung erfolgen, sofern rechtliche Veränderungen nötig sind, ist eine Rückmeldung innerhalb von sechs Monaten vorgesehen (Office of the Plenipotentiary of the Government of the Slovak Republic for the Development of the Civil Society, 2017, S. 6).

Ein weiterer Bestandteil im NAP 2017-2019 ist die Erarbeitung von Schulungsmaterialien (Handbücher, Methoden, e-Learning) in Zusammenarbeit mit den Ministerien für Bildung, Wissenschaft, Forschung und Sport, um so die Beteiligung der Öffentlichkeit zu erhöhen (Open Contracting Partnership, 2019h).

\subsubsection{Ergebnisse der slowakischen Maßnahmen}

Im Ergebnis wurden vom 01. Januar 2011 bis Oktober 2019 fast zwei Millionen Verträge im zentralen Vertragsregister https://www.crz.gov.sk/ veröffentlicht. Diese Verträge (sowohl auf nationaler wie auch auf europäischer Ebene) wurden

\footnotetext{
${ }^{6}$ Seit 2009 hat TI Slowakei ein Online-Portal basierend auf öffentlichen Einkaufsdaten aufgebaut (tenders.sme.sk). Das Portal visualisiert öffentliche Einkäufe nach Einkäufern, Lieferanten, Sektoren und Regionen und bietet herunterladbare, strukturierte Daten an. So konnten sehr viel umfangreichere Analysen durchgeführt werden (Furnas, 2013).
} 
von Ministerien, zentralen staatlichen Verwaltungsstellen, öffentlichen Einrichtungen und nachgeordneten Organisationen dieser Einrichtungen ab dem 01. Januar 2011 geschlossen. Wie erwähnt, tritt ein solcher Vertrag gemäß Dekret 546/2010 erst am Tag nach seiner Veröffentlichung im zentralen Vertragsregister in Kraft. Die Verträge sind ungeschwärzt als PDF-Dokument einsehbar und herunterladbar. Maschinenlesbare Daten sind allerdings nicht verfügbar (Government Office of the Slovak Republic, 2019). Des Weiteren hat sich die Anzahl der Online-Ausschreibungen von $2 \%$ auf über $50 \%$ erhöht und die Anzahl der Bieter von 1,6 auf 3,7 pro Ausschreibung (Šípoš \& Turček, 2015, S. 4). Vor dem neuen Transparenzgesetz im Jahr 2011 schöpften weniger als 5\% der Bevölkerung ihre informationsfreiheitlichen Rechte aus; im ersten Jahr danach nutzten $11 \%$ der Bürger das zentrale Vertragsregister (demnach doppelt so viele), $8 \%$ im Durchschnitt in den Jahren danach. Das bedeutet, dass sich die Öffentlichkeit viel stärker engagiert, wenn die Daten proaktiv bereitstehen im Vergleich zur passiven Datenabholung. Des Weiteren ist der Datenjournalismus präsenter geworden: Die Anzahl der berichteten Fälle zum öffentlichen Einkauf stieg um $25 \%$ in den vier Jahren nach der verpflichtenden Veröffentlichung zwischen 2011 und 2014 (Šípoš et al., 2015, S. 11). Die Besucherstatistiken der Vertragsportale (https://www.crz.gov. sk/ und http://www.otvorenezmluvy.sk/) spiegeln wider, dass beide zusammen im Jahr 2014 etwa 54000 Besuche pro Monat verzeichneten, ein Drittel mehr als in 2012 (Š́poš et al., 2015, S. 8 f.). Darüber hinaus stieg die Geschäftsentwicklung in diesem Umfeld an, da Firmen ihre Dienste zur Datenauswertung und -aggregation anboten (Šípoš et al., 2015, S. 11).

Allerdings besteht weiterer Handlungsbedarf. Öffentliche Einkaufsdaten werden noch nicht mit anderen Daten kombiniert (Furnas, 2013), wenngleich es über Digiwhist erste Ansätze gibt (Government Transparency Institute (GTI), 2019). Die Konsequenzen falschen Handels müssen von außen wahrnehmbar werden, da sie sonst nicht die Glaubwürdigkeit in Staat und Regierung verstärken (Furnas, 2013) 7 . So bestätigte das Interview, dass zumeist interne Sanktionen

${ }^{7}$ Die Lehrer hatten Informationen über die Beschaffung von Blumen durch das Bildungsministerium in Höhe von 9.900 Euro und einen Vertrag über 36 Flaschen Alkohol für je 132 Euro eingereicht. Diese kleinen Beispiele zeichnen ein Bild einer Art kontextualisierter, öffentlicher und partizipatorischer Kontrolle, die ohne Online-Veröffentlichung nicht möglich gewesen wäre. Schließlich führte das Finanzministerium eine Prüfung der Beschaffungstätigkeit des Bildungsministeriums durch. Nach Angaben des „Slovak Spectator“ stellte man fest, dass das Bildungsministerium ,einen schweren Verstoß gegen die Vergaberegeln begangen hat", schlug jedoch keine andere Möglichkeit vor, als dass der Bildungsminister Čaplovič „,solche Probleme künftig vermeiden“ sollte (Vilikovská, 2013). 
greifen, indem beispielsweise Mitarbeiter ausgetauscht würden. Dies sei zwar „gesichtswahrend“, führe allerdings nicht dazu, dass Konsequenzen nach außen sichtbar werden. ${ }^{8}$ Die Datenqualität muss weiter erhöht werden - momentan fehlt bei $10 \%$ aller veröffentlichten Verträge mindestens eine verbindliche Information (Clare et al., 2016). Des Weiteren könnte es sinnvoll sein, den OCDS der OCP zu nutzen, um den Radius der veröffentlichten Daten zu erweitern sowie den Prozess weiter zu strukturieren (Open Contracting Partnership, 2019j). Es hat sich darüber hinaus herausgestellt, dass es für einige Bereiche nicht sinnvoll war, alle Verträge und Dokumente zu veröffentlichen, da die entstehenden Kosten den Nutzen überstiegen. Für diese wurden Ausnahmen eingeführt, zum Beispiel für Friedhöfe, Wasserbetriebe, Studentenwohnheime (Clare et al., 2016). Weitere Ausnahmen betreffen staatliche Unternehmen, die nur die Verträge außerhalb ihres Kerngeschäftes veröffentlichen müssen, sowie öffentliche Sendeanstalten, die ihre Künstlerverträge nicht veröffentlichen müssen (Šípoš, 2015).

Für den Zeitraum 2017 bis 2019 wurde ein weiterer NAP mit insgesamt 68 Maßnahmen erarbeitet, welcher sich zum Zeitpunkt der Erstellung vorliegender Arbeit noch in Umsetzung befindet. Fünf Expertengruppen befassen sich mit den Themenfeldern offene Daten/Transparenz, Partizipation, offene Bildung und Forschung, offene Rechtsprechung und Software-Applikationen. Eine übergreifende Arbeitsgruppe, bestehend aus Vertretern verschiedener Behörden, koordiniert die Expertengruppen (Office of the Plenipotentiary of the Government of the Slovak Republic for the Development of the Civil Society, 2017, S. $5 \mathrm{ff}$.).

Versuche, die Veröffentlichungspflicht bei öffentlichen Verträgen zu umgehen, möchte man künftig durch ein Kontrollsystem unterbinden, mit welchem die Pflicht zur Veröffentlichung besser überprüft werden kann. Die Veröffentlichungspflicht soll von fünf auf zehn Jahre ausgeweitet und den Kommunen die Möglichkeit der Veröffentlichung der Verträge in der zentralen Vertragsdatenbank ermöglicht werden (Office of the Plenipotentiary of the Government of the Slovak Republic for the Development of the Civil Society, 2017, S. 15). Im Zwischenbericht aus dem Jahr 2018 wurden entsprechende Fortschritte bestätigt (Office of the Plenipotentiary of the Government of the Slovak Republic for the Development of the Civil Society, 2018, S. 37 f.). Es wurde ein Vorschlag zur Änderung des Dekrets 211/2000 eingereicht, welcher unter anderem folgende Vorschläge zur Anpassung beinhaltet:

${ }^{8}$ Persönliches Experteninterview mit Isabelle Adam (GTI Ungarn) am 07. März 2019. 
Öffentliche Unternehmen haben keine Möglichkeit, die Veröffentlichungspflicht zu umgehen, sie werden nun verpflichtend in das Gesetz aufgenommen. Gleiches betrifft Quasi-Monopole, zum Beispiel öffentliche Unternehmen, die die Erhebung der Autobahngebühren sicherstellen oder den öffentlichen Transport organisieren. Eine weitere Neuerung ist die Einführung einer Klage, wenn ein Vertrag trotz Verpflichtung nicht veröffentlicht wurde. Die Klage kann dazu führen, dass der Auftragnehmer die Leistung zurückzahlen muss. Im zentralen Vertragsregister verdoppelt sich die Bereitstellungspflicht der Verträge von fünf auf zehn Jahre. Im System werden Funktionen eingerichtet, die Manipulationen an Verträgen sichtbar machen. Kleinen Kommunen wird die Möglichkeit gegeben, das zentrale Vertragsregister ebenfalls für ihre Vertragsveröffentlichungen zu nutzen, um zu vermeiden, dass sie eigene Portale erstellen müssen. Abschließend wird nochmals unterstrichen, dass Fachleute vorab die Bereitstellung der Informationen auf personenbezogene Daten, Geschäftsgeheimnisse, Verschlusssachen etc. überprüfen. Somit sollen die Personen, die Daten veröffentlichen und die in der Regel keine Experten, sondern Laien sind, vor Irrtümern geschützt werden (Ministerstvo Spravodlivosti Slovenskej Republiky, 2017).

\subsubsection{Die Maßnahmen der Slowakei als Best Practices}

Nach vielen Korruptionsskandalen hat sich die Slowakei 2011 zu einem harten Durchgreifen entschieden. Im Zentrum steht eine klare Gesetzgebung, ergänzt um flankierende Maßnahmen im Sinne einer Öffnung des öffentlichen Einkaufs. Diese Maßnahmen zielen auf die Veränderung der öffentlichen Einkaufsorganisation hin $\mathrm{zu}$ stärkerer Zentralisierung, auf eine intensive Interaktion zwischen Vertretern der Zivilgesellschaft und des Staates sowie auf die Nutzung der IKT als Treiber. Mit fast zwei Millionen veröffentlichten Verträgen von 2011 bis 2019 für den Oberschwellen- und Unterschwellenbereich konnten sichtbare Erfolge erzielt werden:

„The Slovak experience shows that a shift to proactive publishing systems as well as more open and transparent procedures - especially in electronic methods - can significantly repel the misuse of public funds, and may also lead to more effective and cheaper procurements" (Budapest Institute for Policy Analysis Ltd., 2012, S. 25).

Die Erhöhung der Datenqualität sowie Notwendigkeit von Schulungen, aber auch systemische Kontrollen zur Einhaltung der Veröffentlichungsvorgaben ergeben sich naturgemäß als Aufgaben, die im Zuge einer fortschreitenden Umsetzung und Weiterentwicklung zu bewältigen sind. Perspektivisch kann es für die 
Slowakei sinnvoll sein, den OCDS zu nutzen und alle Phasen des Einkaufs einzubeziehen. Als zukünftige Chancen können die Entwicklung hin zu LOD angesehen werden und der Nachweis, dass Einkaufspreise tatsächlich sinken und der Allgemeinheit zugutekommen. Ein zu reduzierendes Risiko ist, dass sich Sanktionierungen auf den Innenbereich verlagern, sodass sie für die Öffentlichkeit nicht mehr sichtbar sind und damit unwirksam werden.

Abbildung 9.1 zeigt abschließend eine SWOT-Analyse für die Slowakei.

\section{Stärken}

- Starke Reformen im Jahr 2011 einhergehend mit starker Zentralisierung des öffentlichen Einkaufs und Verpflichtung zur Veröffentlichung aller Verträge

- Wettbewerbszunahme

- Stärkere Sensibilisierung der Öffentlichkeit durch Medienkommunikation

- Anstieg des Datenjournalismus und Auswertung von Verträgen

- Hohe Frequentierung der Webseiten mit veröffentlichten Verträgen zeigen ein hohes öffentliches Interesse

- Starke Zusammenarbeit zwischen NGOs und Vertretern des Staates

- Moderate Kosten für die Softwareeinführung und -wartung

\section{Chancen}

- Erweiterung bestehender Daten um Vernetzung mit anderen Daten, basierend auf dem Beispiel Digiwhist

- Ausweitung der Öffnung auf die Prozessphasen „Planung“ und „Ausführung“

- Verbesserte Wahrnehmung, dass Korruption geringer wird

- Preissenkungen bzw. effizientere Verwendung der Steuermittel

\section{Schwächen}

- Erhöhung der Datenqualität und -vollständigkeit ist nötig

- Fortbildung rund um Daten (Qualität, Datenanalyse) für die Bereitsteller und Nutzer ist erforderlich

- Anwendung internationaler Standards wie z. B. OCDS ggf. sinnvoll zur wieteren Strukturierung und Verbreiterung

- Kosten-Nutzen-Bewertung: In manchen Bereichen war es nicht sinnvoll, alle Verträge zu veröffentlichen, da die Kosten den Nutzen überstiegen (z. B. Friedhöfe)

- Sicherstellung der Einhaltung der verbindlichen Vorgaben ist nicht flächendeckend möglich mangels Ressourcen

\section{Risiken}

- Geringe personelle Kapazität im „Office of Public Procurement“", um z. B. die Ausführung der Verträge zu verfolgen

- Sichtbare Konsequenzen an der Außenkante fehlen - eher Verlagerung auf interne Sanktionierung (Fluktuation Mitarbeiter). Dies kann ggf. die Glaubwürdigkeit bei Bürgern reduzieren, da rein interne Sanktionen nicht sichtbar sind

Abbildung 9.1 SWOT-Analyse Slowakei 


\subsubsection{Frankreich}

\subsubsection{Dezentralisierung und Fragmentierung}

Frankreich verfügt (Stand Dezember 2018) über ein BIP von 2,35 Billionen Euro (Urmersbach, 2019) und ein öffentliches Ausschreibungsvolumen von etwa 200 Milliarden Euro, von denen $95 \%$ oberhalb von 25.000 Euro liegen (Dingremont, 2018, S. 2). Etwa $18 \%$ der Ausschreibungen laufen über die TED und sind damit dem Oberschwellenbereich zuzuordnen, $82 \%$ der Ausschreibungen betreffen demzufolge den Unterschwellenbereich (European Commission, 2016b, S. 76). Im Bereich des öffentlichen Einkaufs bejahen $50 \%$ der befragten Unternehmen gemäß Flash Eurobarometer aus 2014, dass Korruption auf der nationalen Ebene erfolgt, $56 \%$ bejahen dies für die regionale/ lokale Ebene (European Commission, 2014, S. 74 f.). Frankreich belegt beim TI Korruptionsindex Platz 21 mit 72 Punkten von insgesamt 100 Punkten. Dieser Wert unterlag in den letzten zehn Jahren nur geringen Schwankungen (Transparency International e. V., 2019b).

Frankreich ist politisch mit 18 Regionen als „regionalisierter Staat“ einzustufen (Berge \& Grasse, 2004, S. 73; Institut national de la statistique et des études économiques (Insee), 2019). Es agiert dezentral, aber nicht föderal. So existiert zum Beispiel kein Zwei-Kammern-System und die Regionen haben keine Gesetzgebungskompetenzen, dennoch besitzen sie Befugnisse zur regionalen Wirtschaftsentwicklung oder Verkehrsplanung (Vogel, 2005).

Für die Vergabe bedeutet dies, dass es aufgrund der Dezentralisierung eine hohe Anzahl von Aufsichtsbehörden, aber auch eine hohe Anzahl von Ausschreibungen gibt: „France stands out as having the highest number of procurement procedures per year within the EU. The majority of these contracts are public works carried out at the local and regional levels" (European Commission, 2016b, S. 76).

\subsubsection{Die komplexe Regulierung des öffentlichen Einkaufs}

Frankreich verfügt als eines der Länder der ersten Stunde über ein Informationsfreiheitsgesetz (Gesetz Nr. 78-753), das bereits 1978 in Kraft trat (University College London (UCL), 2019). Der „Code des Marchés Publics“ (CMP) aus dem Jahr 2006 (Dekret Nr. 2006-975) integrierte zum ersten Mal das EU-Recht (2004/17/ EC und 2004/18/EC) in nationales Recht (European Commission, 2016b, S. 76). Der frühere Art. 133 (Dekret Nr. 2006-975) (Secrétariat général du Gouvernement, 2016a) verpflichtete die Einkäufer, eine Liste der im Vorjahr abgeschlossenen Verträge zu veröffentlichen. Allerdings waren die meist verteilt veröffentlichten Daten nicht standardisiert, sodass sowohl der Zugang als auch die Auswertung für Bürger 
schwierig war. Um ein Ökosystem öffentlicher Auftragsdaten zu erzeugen, war eine Standardisierung erforderlich. Es folgten diverse Verordnungen mit Detaillierungen, die später aufgehoben oder modifiziert wurden.

Der Open Data-Vorstoß gelang schließlich im Jahr 2016 durch das „,Gesetz für eine digitale Republik“ (Secrétariat général du Gouvernement, 2016b). Es ist ein umfassendes Gesetz, das Datenschutz, Informationsrechte und Open Data berücksichtigt (Proust \& Goossens, 2016). Das Dekret vom 14. April 2017 (Secrétariat général du Gouvernement, 2017) spezifizierte darauf folgend die Daten und Formate, die auf den Einkäuferprofilen für reguläre Verträge, aber auch für Verträge rund um Verteidigung und Sicherheit sowie Konzessionen hinterlegt werden müssen (Tales, 2019; Wickberg, 2018, S. 30). Unterhalb von 25.000 Euro Vertragsvolumen gibt es eine Freistellungsschwelle.

Die wesentlichen aktuellen Regeln zum öffentlichen Einkauf stehen in der Verordnung 2018-1074 und dem Dekret 2018-1075 (Secrétariat général du Gouvernement, 2018). Hiermit wurde in einer konsolidierenden Kodifizierung versucht, die zuvor sehr unterschiedlichen Gesetzestexte zusammenzuführen. Im Ergebnis entstand ein Gesetz mit 1747 Artikeln (Brenot et al., 2019). Die allgemeinen Grundsätze orientieren sich an jenen der EU-Rechtsprechung: Gleichbehandlung zwischen Wettbewerbern, freier Zugang zu Aufträgen, Transparenz, Wirtschaftlichkeit und Effizienz (Verordnung 2018-1075, Art. L.3). Dies zeigt bereits, wie komplex der öffentliche Einkauf in Frankreich reguliert ist.

Frankreich hat die Öffnung des öffentlichen Einkaufs parallel zur Anpassung der Rechtsprechung in Aktivitäten mit Nichtregierungsorganisationen verankert. So wird im ersten NAP 2015 bis 2017 und in seiner Fortführung im zweiten NAP 2018 bis 2020 deutlich die Absichtserklärung Nr. 2 „Increasing transparency in public procurement" benannt (République Francaise, 2017, S. 9). Darüber hinaus ist Frankreich seit 2014 Mitglied in der OGP und dort seit 2015 Mitglied im Lenkungsausschuss. Von 2016 bis 2017 war Frankreich Co-Präsident. Darüber hinaus partizipiert Frankreich in der OCP. Es nutzt zwar den OCDS noch nicht (Open Contracting Partnership, 2019j), hat jedoch eine Übersetzung des Standards ins Französische vorgenommen. Als eines von fünf Gründungsmitgliedern ist es seit 2016 Teil der C5-Initiative und hat dort seit 2018 den Vorsitz inne (Contracting Five, 2019b), allerdings gibt es hierzu keine veröffentlichten Aktivitäten (siehe auch Abschnitt 5.2.4.7). Darüber hinaus engagiert sich Frankreich im Digiwhist-Projekt (Government Transparency Institute (GTI), 2019).

\subsubsection{Die Öffnung des öffentlichen Einkaufs in Frankreich}

Das Finanz- und Wirtschaftsministerium ist hauptverantwortlich für den rechtlichen Rahmen der öffentlichen Vergabe. Darüber hinaus ist eine Institution namens „Etalab“ ein weiterer wichtiger Akteur zur Öffnung der Einkaufsdaten. 
Etalab ist Teil der interministeriellen Direktion „Digitales und Informationssysteme/Kommunikation des Staates (DINSIC)“. Es ist unter anderem zuständig für die Entwicklung und den Betrieb des Open Data-Portals https://www.data. gouv.fr (Direction interministérielle du numérique et du système d'information et de communication de l'Etat, 2019b). ${ }^{9}$

Der Einkauf erfolgt in den Bereichen Dienstleistungen, Güter und Bauvorhaben. Für den Oberschwellenbereich gelten die EU-Regularien (forum vergabe e. V., 2017) - sofern die EU-Wertgrenzen erreicht werden, muss ein formalisiertes Ausschreibungsverfahren angewendet werden (Dekret 2018-1075, Kap. 4). Die nationalen Wertgrenzen sind in Abbildung 9.2 dargestellt.

\begin{tabular}{|c|c|c|c|c|}
\hline Kategorien & \multicolumn{3}{|c|}{ Nationale Vergaben } & EU-Vergaben \\
\hline $\begin{array}{l}\text { Art der } \\
\text { Vergabe }\end{array}$ & $\begin{array}{l}\text { Direktvergabe } \\
\text { für Produkte } \\
\text { und Dienstleis- } \\
\text { tungen }\end{array}$ & $\begin{array}{l}\text { Freie Form oder } \\
\text { angepasste Ver- } \\
\text { öffentlichungen }\end{array}$ & $\begin{array}{l}\text { Nationale Aus- } \\
\text { schreibungen } \\
\text { (Veröffent- } \\
\text { lichung im } \\
\text { BOAMP oder } \\
\text { JAL) }\end{array}$ & $\begin{array}{l}\text { EU-relevante } \\
\text { Verträge (Ver- } \\
\text { öffentlichung } \\
\text { im BOAMP } \\
\text { und OJEU) }\end{array}$ \\
\hline $\begin{array}{l}\text { Zentrale } \\
\text { Vergabe- } \\
\text { stellen inkl. } \\
\text { Universi- } \\
\text { täten }\end{array}$ & 25.000 Euro & $\begin{array}{l}25.000 \text { bis } \\
89.999,99 \text { Euro } \\
\text { (allgemein) oder } \\
25.000 \text { bis } \\
144.000 \text { Euro } \\
\text { (Ausnahmerege- } \\
\text { lung für öffentli- } \\
\text { che Einrichtungen } \\
\text { mit Forschungs- } \\
\text { auftrag. Optionale } \\
\text { Verwendung der } \\
\text { im Dekret vorge- } \\
\text { schriebenen for- } \\
\text { malisierten Ver- } \\
\text { fahren) }\end{array}$ & $\begin{array}{l}90.000 \text { bis } \\
143.999,99 \\
\text { Euro (Produkte } \\
\text { und Dienstleis- } \\
\text { tungen); } 90.000 \\
\text { bis } \\
5.547 .999,99 \\
\text { Euro (Bauleis- } \\
\text { tungen) } \\
\text { (Verpflichtende } \\
\text { Nutzung forma- } \\
\text { lisierter Prozes- } \\
\text { se wie im De- } \\
\text { kret beschrie- } \\
\text { ben) }\end{array}$ & $\begin{array}{l}\text { 5.548.999 Euro } \\
\text { (Bauleistun- } \\
\text { gen); } 144.000 \\
\text { Euro (Produkte } \\
\text { und Dienstleis- } \\
\text { tungen) }\end{array}$ \\
\hline
\end{tabular}

Abbildung 9.2 Nationale Wertgrenzen Frankreich (in Anlehnung an Estermann \& Kupriyanova, 2018, S. 35, übersetzt durch Verfasserin)

\footnotetext{
${ }^{9}$ Weitere Aufgabenbereiche: Schaffung von Transparenz öffentlicher Maßnahmen, Beteiligung der Öffentlichkeit und Koproduktion mit der Gesellschaft, soziale Innovationen, Stärkung des Vertrauens der Bürger, verbesserte Verwaltungsfunktionen, Beitrag zum internationalen Einfluss Frankreichs.
} 
Unter 25.000 Euro (exklusive Mehrwertsteuer) können Verträge ohne vorherige Bekanntmachung und Wettbewerb (Dekret 2018-1075, Kap. 2, Art. R2122-8) vergeben werden. Darüber hinaus gelten weitere Ausnahmen, zum Beispiel für Verträge über Dienstleistungen mit dem Gewinner eines Wettbewerbs (Dekret 2018-1075, Kap. 2, Art. R2122-6) oder für Forschungs- oder Experimentierzwecke (Dekret 2018-1075, Kap. 2, Art. R2122-10). Die Auftraggeber werden aufgefordert, die Verträge nicht systematisch an die gleichen Wirtschaftsteilnehmer zu vergeben (Law Business Research Ltd., 2019).

Oberhalb von 25.000 Euro und unterhalb der EU-Schwellenwerte können „angepasste“ Verfahren (,procedures adaptées“) anwendet werden (Dekret 2018-1075, Kap. 3). Bei Verfahren zwischen 25.000 Euro und bis 90.000 Euro (exklusive Mehrwertsteuer) können die Ausschreibungsstellen selbst wählen, in welchem Umfang sie veröffentlichen möchten oder welche genauen „Terms and Conditions of Procedure" sie wählen (Holterbach \& Dubrulle, 2019).

Bei Vertragsvolumina ab 90.000 Euro (exklusive Mehrwertsteuer) ist eine Veröffentlichung im Bulletin officiel des annonces de marchés publics (BOAMP) über https://www.boamp.fr oder in einer anderen zugelassenen Zeitung (Dekret 2018-1075, Kap. 5, Art. R2131-12) zwingend. Zu den anderen berechtigten Zeitungen zählen zum Beispiel das „Journal d'annonces légales“ (JAL), aber auch Tages- und Lokalzeitungen (zum Beispiel „Republic Lorrain“ im Großraum Metz).

Die EU Richtlinie 2014/24 sieht E-Procurement verbindlich für alle Verträge im Oberschwellenbereich vor. Dies wurde in nationales Recht umgesetzt (Dekret 2018-1075, Kap. 2, Art. 2132-7). Demnach müssen alle Ausschreibungen über 25.000 Euro (exklusive Mehrwertsteuer) ab 01. Oktober 2018 elektronisch publiziert werden müssen. Eine Ausnahme sind unter anderem Ausschreibungen, die unterhalb der Schwellenwerte des formalisierten Verfahrens liegen (Dekret 2018-1075, Kap. 2, Art. 2132-12, Abs. 1) oder Ausschreibungen, die mit elektronischen Mitteln nicht sicher abgewickelt werden könnten (Dekret 20181075, Kap. 2, Art. 2132-12, Abs. 7).

Alle Aufträge über 25.000 Euro (exklusive Mehrwertsteuer) müssen ab 01. Oktober 2018 (Dekret 2018-1075, Kap. 2, Art. R2132-2 ff.) mit Käuferprofilen über eine elektronische Plattform abgewickelt werden. Dies war zwar zuvor bereits für Ausschreibungen ab 90.000 Euro verpflichtend (Secrétariat GénéralService de la Communication Ministère de l'Économie et des Finances, 2018), wurde aber nun nochmals verstärkt. 
Die minimal in Form von JSON oder XML bereitzustellenden Daten sind:

- Identität (ID)

- Système d'identification du répertoire des établissements (SIRET)-ID und Name des öffentlichen Einkäufers

- SIRET-ID und Name der Firma

- Datum der Notifikation

- Datum der Veröffentlichung der Daten

- Vertragsgegenstand

- Vertragssumme exklusive Steuern

- Vertragsdauer (in Monaten)

- CPV-Code

- Vertragserfüllungsort (INSEE-Code oder postalischer Code)

- Art des Vertrags

- Verfahrensart

- Art des Preises (zum Beispiel fest, nach Aufwand)

- Anpassungen der Dauer, Beträge oder Firmen

Des Weiteren sind auf https://www.data.gouv.fr Vertragsdaten von 2011 bis aktuell 2017 in einer Excel-Tabelle hinterlegt (Direction interministérielle du numérique et du système d'information et de communication de l'Etat, 2019c). Diese spiegeln zwar nicht den Gesamtwert des öffentlichen Einkaufs wider ${ }^{10}$, zeigen jedoch einen Ansatz, wie eine solche vereinfachte Darstellung aussehen kann. Die Darstellung orientiert sich an den minimalen Veröffentlichungspflichten: das Berichtsjahr, der öffentliche Auftraggeber, die Beschaffungsstelle, der Auftragnehmer, das SIRET-Attribut, das Veröffentlichungsdatum, die Postleitzahl, die Stadt, Einkaufskategorie, Vertragsgegenstand, Budget, Betrag und ob es sich um ein KMU handelt (Direction interministérielle du numérique et du

\footnotetext{
${ }^{10}$ Das Beispiel aus dem Jahr 2013 addiert nur etwa 6,6 Milliarden Euro über etwa 14000 Zeilen (Direction interministérielle du numérique et du système d'information et de communication de l'Etat, 2019c). Der öffentliche Einkauf wird allerdings auf 200 Milliarden Euro geschätzt (Dingremont, 2018).
} 
système d'information et de communication de l'Etat, 2014). Diese Daten werden mit dem Open Data-Portal der französischen Regierung verknüpft. Hierüber ist die Visualisierung und Auswertung historischer und aktueller Ausschreibungen möglich (Direction interministérielle du numérique et du système d'information et de communication de l'Etat, 2019a).

Flankiert werden diese Bemühungen von einer starken Vereinfachung der Angebotsabgabe mit Beschluss aus dem Jahr 2017: Die Firmen erhalten eine SIRET-Nummer, die für die Ausschreibungsstelle ausreicht, um alle notwendigen Informationen zu bekommen. Des Weiteren muss hierfür das Käuferprofil der ausschreibenden Stelle erstellt werden (eigenes Datenbanksystem im Hintergrund). Rechnungen können von Unternehmen an Auftraggeber über die Nutzung eines speziellen Zahlungsportals namens „Chorus Pro“ vollständig elektronisch übermittelt werden (Hartmann, 2017) (Verordnung Nr. 2014-697).

Im ersten NAP 2015 bis 2017 findet sich, wie bereits erwähnt, die Öffnung des öffentlichen Einkaufs als Maßnahme. Sie wird in Bezug auf ihre potentielle ökonomische Auswirkung als „transformativ“ eingeordnet (andere Kategorien sind „keine“, „minimal“ und „moderat“). Gegenstand der Maßnahme sind die Standardisierung der Datenformate für alle öffentlichen Ausschreibungen, um potentiellen Bietern den Zugang zu vereinfachen, die Veröffentlichung der Daten des BOAMPs und der Käuferprofile sowie eine Verstärkung der Offenlegung vergebener öffentlicher Ausschreibungen (Verpflichtung für den Oberschwellenbereich, Freiwilligkeit für den Unterschwellenbereich). In den entsprechenden Verträgen sollen künftig Klauseln enthalten sein, die die Öffnung der Daten ermöglichen (Wickberg, 2018, S. 28).

Trotz zahlreicher rechtlicher Veränderungen konnten im geplanten Zeitraum die Maßnahmen nicht vollständig umgesetzt werden, sodass diese im zweiten NAP 2018 bis 2020 fortgeführt werden. Zudem befürchtet man, dass sich mangels Kapazität die lokalen Umsetzungen trotz der Bemühungen auf der nationalen Ebene verzögern werden (Wickberg, 2018, S. 31).

Im zweiten NAP 2018 bis 2020 wurden dann die Aktivitäten gemäß Tabelle 9.3 festgelegt. Es wurde unterstrichen, dass vollständige Transparenz im öffentlichen Einkauf nur mit einem intensiven Training und starker Vereinfachung erfolgen kann (République Francaise, 2017, S. 10). Zum jetzigen Zeitpunkt gibt es noch keinen Zwischenbericht der Ergebnisse. 
Tabelle 9.3 Nationaler Aktionsplan 2018 bis 2020 in Frankreich für Maßnahme 2 „Increasing transparency in public procurement“ (in Anlehnung an République Francaise, 2017, S. 10, übersetzt durch Verfasserin)

\begin{tabular}{|c|c|}
\hline Maßnahmen & Zeitleiste \\
\hline $\begin{array}{l}\text { - Design eines ,einzigen Prozesses“ der } \\
\text { Einkaufsdaten }\end{array}$ & März 2017-September 2018 \\
\hline $\begin{array}{l}\text { - Implementierung des Prozesses auf dem Portal data. } \\
\text { gouv.fr }\end{array}$ & April 2018 \\
\hline $\begin{array}{l}\text { - Erarbeitung des Umfangs der Daten, welcher über die } \\
\text { essentiellen Daten gemäß Gesetz hinausgehen soll } \\
\text { - Diese Daten sollen in Kooperation mit Entwicklern, } \\
\text { öffentlichen Einkäufern etc. über Open Labs erarbeitet } \\
\text { werden (das erste Open Lab fand am 25. September } 2017 \\
\text { statt) }\end{array}$ & $\begin{array}{l}\text { Zweites Open Lab in } \\
\text { 2018, anhaltende Arbeit } \\
\text { bis Ende } 2019\end{array}$ \\
\hline $\begin{array}{l}\text { - Entwicklung und Implementierung innovativer } \\
\text { Nutzungsmöglichkeiten öffentlicher Einkaufsdaten im } \\
\text { Rahmen der zwei Testfälle: Bretagne und Okzitanien }\end{array}$ & Ende 2018 \\
\hline $\begin{array}{l}\text { - Verbreitung dieser Praktiken in anderen Regionen: } \\
\text { Integration weiterer Regionen } \\
\text { und Veröffentlichung ihrer Daten im gleichen Format }\end{array}$ & Ab Januar 2018 \\
\hline $\begin{array}{l}\text { - Verbreitung dieser Standards in der internationalen } \\
\text { Gemeinschaft über die C5-Initiative }\end{array}$ & Ab Januar 2018 \\
\hline $\begin{array}{l}\text { - Integration weiterer Länder in der C5-Initiative und } \\
\text { Werbung für die Nutzung internationaler Standards in } \\
\text { diesen Ländern }\end{array}$ & Frühjahr 2018 \\
\hline $\begin{array}{l}\text { - Entwicklung einer Richtlinie für Unterstützung und } \\
\text { Training für die öffentlichen Stellen, die in die digitale } \\
\text { Transformation des öffentlichen Einkaufs involviert sind }\end{array}$ & 2020 \\
\hline
\end{tabular}

Begleitet werden diese Maßnahmen ebenfalls durch Aktivitäten rund um die Beteiligung der Bürger. Frankreichs Bürger haben Zugang zum Portal http:// www.vie-publique.fr. Dieses bietet zentral umfangreiche Informationen rund um das öffentliche Leben, aktuelle Debatten und die Funktionsweise der öffentlichen Institutionen. Seit November 2014 führt dieses Portal auch die Konsultationen und öffentlichen Debatten sowie Zusammenfassungen der Ergebnisse auf, so dass eine einfachere Beteiligung möglich ist (Open Government Partnership, 2019d). 


\subsubsection{Ergebnisse der Maßnahmen Frankreichs}

Frankreich hat in den vergangenen Jahren diverse Anpassungen seiner Rechtsprechung vorgenommen. Änderungen der Inhalte zielen auf eine stärkere Offenlegung öffentlicher Einkaufsdaten - die Vereinfachung der Rechtsstruktur zielt auf stärkere Konsolidierung. Das Land engagiert sich stark in den Nichtregierungsorganisationen OGP und OCP. Ab Oktober 2018 wurde die Veröffentlichung zu Vergaben über 25.000 Euro (exklusive Mehrwertsteuer) rechtsverbindlich. Des Weiteren kann die Bereitstellung von bestimmten Vertragsdaten auf data.gouv.fr für den Zeitraum 2011 bis 2017 als erster Erfolg gewertet werden. Frankreich forciert E-Procurement-Lösungen stark, zum Beispiel über die Nutzung des Käuferprofils, was nicht nur für eine Vereinfachung der Ausschreibungs- und Vergabeverfahren, sondern gleichzeitig für mehr Transparenz sorgt. Interessante organisatorische Lösungen sind die Schaffung der DINSIC für intersektorale Herausforderungen und die dort angesiedelte Organisationseinheit Etalab sowie die Nutzung eines „General Data Administrators“. Hierüber werden klare Zuständigkeiten für die Förderung der beabsichtigten und ernstgemeinten digitalen Transformation des öffentlichen Einkaufs geschaffen.

Aktuell nicht bekannt sind die Ergebnisse in Bezug auf die Nutzung der bereits veröffentlichten Einkaufsdaten sowie ihre Effekte auf Einsparungen, bessere Preise oder eine erhöhte Lieferqualität. Vertiefend müsste man ebenfalls verstehen, wie die Daten aggregiert werden (Prozess, Datenbereitstellung, Datenqualität) (Tales, 2019). Die Freiwilligkeit zur Öffnung öffentlicher Einkaufsdaten im Unterschwellenbereich muss beobachtet und gegebenenfalls rechtsverbindlich gestaltet werden. Hierzu wären weitere, begleitende Erhebungen hilfreich.

\subsubsection{Die Maßnahmen Frankreichs als Best Practices}

Die Situation in Frankreich ist geprägt von einem sehr komplexen institutionellen Arrangement mit einem umfänglichen Gesetzesrahmen. Im Hinblick auf Open Contracting nimmt Frankreich durch den NAP und das Mitwirken in diversen Organisationen die Rolle eines Vorreiters ein: Es erklärt sich verbindlich zur Schaffung von Transparenz im öffentlichen Einkauf bereit und verpflichtet sich zur Bereitstellung der Informationen in maschinenlesbaren Formaten.

Die Ausführungen zeigen allerdings auch, dass bereits die Standardisierung der öffentlichen Einkaufsdaten (allein für den Oberschwellenbereich) mehr Zeit als gewünscht in Anspruch nimmt. Das bedeutet, dass trotz einer guten strategischen Verankerung und Anpassung des rechtlichen Rahmens die Umsetzung eine besondere Herausforderung darstellt. Somit verbleibt das Risiko, dass sich Ergebnisse verzögern und sich das aktuelle Momentum reduziert. 
Ergänzend zu den bereits zuvor geschilderten Stärken und Schwächen könnte Frankreich perspektivisch als weitere Chance die Verknüpfung der vorhandenen öffentlichen Einkaufsdaten mit anderen Regierungsdaten aufgreifen sowie die Darlegung der Einkaufsdaten auch auf andere Teilprozesse wie Planung und Ausführung ausweiten. Dies scheint allerdings erst dann sinnvoll, wenn eine Routine bei den Prozessen zur Öffnung der öffentlichen Einkaufsdaten eingekehrt ist. Abbildung 9.3 zeigt abschließend die SWOT-Analyse für Frankreich.

\section{Stärken}

- Seit dem Jahr 1978 verfügt Frankreich über ein Informationsfreiheitsgesetz - der Zugang zu amtlichen Informationen ist ein hohes Gut in Frankreich

- Klares politisches Engagement in punkto „Offene Daten“ und „Offene öffentliche Einkaufsdaten“"

- Gesetzliche Verpflichtung zur Darlegung detaillierter Daten ab 25.000 € Vertragsvolumen exklusive Mehrwertsteuer und Nutzung von Käuferprofilen

- Veröffentlichung einer Vielzahl von Vertragsdaten auf dem zentralen Open Government Portal ,www.gouv.france.fr für die Jahre 2011-2017

- Flankierende Maßnahmen rund um Standardisierung und E-Procurement (z. B. elektronische Rechnung)

- Interessante organisatorische Lösungen (DINSIC, ,General Data Administrator“)

\section{Chancen}

- Trennung zwischen dem Umfang gesetzlicher Veröffentlichungspflichten im Rahmen des Vergabeprozesses und im Rahmen von Open Data

- Ausweitung auf den Unterschwellenbereich, auf die Phasen „Planung“ und „Ausführung“" sowie Darlegung kompletter Verträge

- Vernetzung bestehender Daten mit anderen Daten, basierend auf dem Beispiel Digiwhist

\section{Schwächen}

- Es werden zwar Vertragsdaten veröffentlicht, jedoch keine gesamthaften Verträge

- Freiwilligkeit der Veröffentlichung für den Unterschwellenbereich; Pflicht beschränkt sich auf den Oberschwellenbereich

- Es gibt kaum/keine Berichte zu Erfahrungen rund um die Auswertung dieser Daten, weiters gibt es kaum Zusammenhangsanalysen zur Korruption

- Inwieweit die veröffentlichten Daten vollständig und qualitativ hochwertig sind, bleibt unklar

\section{Risiken}

- Beherrschbarkeit der institutionellen Komplexität

- Weitere Standardisierung als Voraussetzung für die weitere Öffnung der öffentlichen Einkaufsdaten

Abbildung 9.3 SWOT-Analyse Frankreich 


\subsubsection{Vergleich der Länderbeispiele und Implikationen für Deutschland}

Die genannten Länderbeispiele zeigen, wie die Öffnung des öffentlichen Einkaufs erfolgen kann. Beide Länder haben trotz ihrer Unterschiede in Bevölkerungszahl, BIP und öffentlichem Einkaufsvolumen in den letzten fünf bis acht Jahren deutliche Fortschritte erzielt. Auf Gemeinsamkeiten und Unterschiede soll nun abschließend eingegangen werden.

\section{Gemeinsamkeiten}

Beiden Ländern gemeinsam ist zunächst eine schriftlich niedergelegte politische Zusage, diese Öffnung, auch als Teil der E-Government-Strategie, verbindlich umsetzen zu wollen (European Commission, 2015a, S. 8 ff.; 2016a, S. 14 ff.). Vergleichbar ist auch der Ansatz der Standardisierung und Zentralisierung, wenngleich auch unterschiedlich (in der Slowakei in Bezug auf den Einkauf, in Frankreich in Bezug auf den Rechtsrahmen). Diese Bestrebungen werden durch zusätzlich gegründete Organisationseinheiten unterstützt (in der Slowakei durch das „Office for Public Procurement“, in Frankreich durch „Etalab“). Eine weitere, signifikante Parallele ist auch die Beteiligung in Nichtregierungsorganisationen (Slowakei seit dem Jahr 2011, Frankreich seit dem Jahr 2014) wie der OGP und OCP. Damit steigt die Selbstverpflichtung zur Umsetzung sowohl durch Formulierung von NAPs als auch infolge der Erwartungshaltung von außen.

\section{Unterschiede}

Frankreich fokussierte zunächst auf die Vereinheitlichung eines sehr komplexen und historisch gewachsenen Rechtsrahmens, während die Slowakei bereits neue Gesetze erlassen konnte und hiervon auch sichtbar Gebrauch gemacht hat. Die Slowakei konnte somit etwas schneller mit der konkreten Umsetzung beginnen, die vermutlich durch eine insgesamt geringere Anzahl öffentlicher Behörden vereinfacht wurde. Die Slowakei zeigt sich deutlich radikaler, indem sie beispielsweise die Gültigkeit der Verträge an deren Veröffentlichung knüpft und Klagen zulässt. Eine fehlende Öffnung kann sogar die Rückzahlung der Leistungen durch den Auftragnehmer nach sich ziehen. Hier erscheint Frankreich gemäßigter und fokussiert zunächst eher auf die Anpassung von Strukturen und Abläufen.

Während die Slowakei bereits vollständige Verträge für den Ober- und Unterschwellenbereich veröffentlicht (allerdings als PDF), macht Frankreich eher selektive Daten öffentlich (als Excel), und zwar bislang nur für den Oberschwellenbereich verbindlich und lediglich freiwillig für den Unterschwellenbereich. Die bereitzustellenden Daten für den Unterschwellenbereich gehen in 
jedem Fall bei beiden Ländern über jene in Deutschland hinaus. Auch im Hinblick auf die Kontrolle messbarer Ergebnisse stellt sich die Slowakei sehr fortschrittlich dar und hat begleitend diverse Indikatoren und Messungen genutzt, um die Wirksamkeit ihrer Maßnahmen zu überprüfen. Insgesamt erscheint die Abstimmung in der Slowakei zwischen Politik, NGOs und Gesellschaft enger, Anpassungen scheinen schneller vorgenommen werden zu können. Dies mag damit zu tun haben, dass die Slowakei von Beginn an Partizipation als wichtiges Element in ihren Maßnahmen integriert und hier auch kurze Antwortund Umsetzungsfristen festgelegt hat. Frankreich setzte hier zunächst auf eine ergänzende Organisationsstruktur (hier Etalab). Dies erscheint in Anbetracht der Größe und Fragmentierung Frankreichs sinnvoll. Mittlerweile hat Frankreich auch Maßnahmen zur Beteiligung der Bürger aufgegriffen.

\section{Differentialdiagramm zur Visualisierung}

Um einen strukturierteren und auch visuellen Vergleich beider Länder zu ermöglichen, wurden basierend auf den vorherigen sieben Wirkungsdimensionen 22 weitergehende Kriterien zur Bewertung gebildet. Die Basis für die Kriterienliste bilden die verwendete Literaturrecherche (u.a. Granickas, 2016; Mendes \& Fazekas, 2018; OECD, 2009b; Open Contracting Partnership, 2019f) und bisher gewonnene Erkenntnisse aus der Dissertation. Die Auswahl der Kriterien ist zugunsten einer ausgewogenen Verteilung über die sieben Dimensionen erfolgt. Somit ist die Kriterienliste keine erschöpfende Darstellung zur generellen Bewertung von Best Practices, sondern eher ein praktisches Prüfschema. Anschließend wurde jedes Kriterium für jedes Land bewertet. Das Ergebnis zeigt Tabelle A.12, die der Übersichtlichkeit halber im Anhang zu finden ist.

Auch das Verlaufsmuster des Differentialdiagramms zeigt noch einmal deutlich, dass sich die Slowakei und Frankreich in den Wirkungsdimensionen Strategie, Organisation und Recht auf ähnlichem Niveau bewegen, aber dabei unterschiedliche Aspekte betonen. Mit Blick auf Transparenz, Partizipation und Kollaboration sind die größten Abweichungen zwischen beiden Ländern zu verzeichnen: Hier erscheint die Slowakei deutlich aktiver als Frankreich. Die geringste Beteiligung ist für beide Länder in der Dimension „Technologie“ zu verzeichnen, da hier die zukunftsgerichteten Technologien noch keinen Einzug erhalten haben. Aus diesem Grund werden in Abschnitt 9.3 ergänzend drei zukunftsweisende Beispiele diesbezüglich vorgestellt. 


\section{Ableitungen für Deutschland}

Für Deutschland ergibt sich daraus eine interessante Beobachtung: Aufgrund seiner Größe und seines föderalen Aufbaus wird Deutschland vermutlich ähnlichen strukturellen Herausforderungen wie Frankreich begegnen, die mit vergleichbaren Maßnahmen gelöst werden könnten. Für eine zügige und schlagkräftige Umsetzung wäre dann eine Orientierung an den Ideen der Slowakei wünschenswert. Eine Kombination klassischer mit agilen Vorgehensweisen bietet sich dabei für eine potentielle Umsetzung an. Insgesamt erscheint es hilfreich, diese Veränderungen für den öffentlichen Einkauf - genauso wie Frankreich - als digitale Transformation einzuordnen. Hiermit wird eine mehrjährige, intensive und vielschichtige Entwicklung anstelle einzelner Aktivitäten angekündigt, was sicherlich angemessen ist und die richtige Erwartungshaltung setzt. Die Implikationen hieraus werden in Kapitel 10 im Leitbild und in den Handlungsempfehlungen konkreter aufgegriffen.

\subsection{Zukunftsweisende technologische Beispiele}

Ergänzend werden in diesem Kapitel drei zukunftsweisende Beispiele vorgestellt, die insbesondere die Kategorien Technologie und Transparenz abdecken und außerdem Preisträger des EU Datathons ${ }^{11}$ waren. Ihre Präsentation wird anhand einer Kurzdarstellung erfolgen.

\section{C4P - Künstliche Intelligenz, Automation und Textmining im öffentlichen Einkauf}

Anbieter der C4P-Plattform ist das gleichnamige Unternehmen, welches im Jahr 2017 gegründet wurde und seinen Sitz in Brüssel hat (C4P, 2017a, S. 2). Die geographische Abdeckung umfasst die EU. Die Zielgruppen sind insbesondere die öffentliche Verwaltung, die Wirtschaft, aber auch die Bürger (C4P, 2017a, S. 6).

C4P bietet unter Nutzung von künstlicher Intelligenz, Machine Learning, Automation und Textmining ${ }^{12}$ Informationen zum öffentlichen Einkauf der EU. Konkrete Anwendungsmöglichkeiten umfassen Informationen zum Einkaufsverhalten, zur aktuellen Leistungsfähigkeit bestehender Unternehmen,

\footnotetext{
${ }^{11}$ „Der EU Datathon 2019 ist ein Wettbewerb, der die Weiterverwendung offener EU-Daten aus dem Offenen Datenportal der EU fördert“ (Europäische Union, 2019a).

${ }^{12}$ Text Mining ist das maschinelle Auslesen unbekannter Informationen aus nicht oder wenig strukturierten Texten (Geierhos, 2018b).
} 
zur Marktsituation, zu den vermutlich besten Firmen für eine Zusammenarbeit sowie Preisinformationen (zum Beispiel das durchschnittliche Preislevel). Über Textmining können passende Ausschreibungen gezielter gefunden werden als über generische und oftmals falsche CPV-Codes. Weiters können passende Ausschreibungen automatisch für eine Weiterqualifizierung über die Plattform an die jeweiligen Einkäufer in den Unternehmen versendet werden. Basierend auf den eigenen Präferenzen kann so eine verbesserte Jahresplanung aufgebaut werden. Über die Marktsegmente, in denen die eigene Organisation tätig ist und in welchem Umfang, sowie neue Marktsegmente können laut C4P Informationen in nur wenigen Minuten zusammengestellt werden (C4P, 2017b).

Aktuell umfasst die Plattform 133 einkaufende Organisationen, 4300 Lieferanten, 23 Sektoren und etwa 3,5 Milliarden Euro Einkaufsvolumen (C4P, 2019). Die Kosten für die Nutzung der Plattform sind auf der Homepage nicht hinterlegt; sie sind angebotsbasiert. Grundsätzlich soll ein Freemium-Modell angeboten werden: Frei soll die Nutzung eines eingeschränkten Umfangs der Plattform für Bürger und Universitäten sein; das Premium-Modell mit vollem Umfang findet dann Anwendung für alle anderen Nutzer (C4P, 2017a, S. 9). C4P war der Gewinner des EU Datathons 2017 (European Union, 2017).

\section{Tenderlake - Smart Assistance für passende öffentliche Ausschreibungen}

Tenderlake Ltd. ist ein Unternehmen, welches im Jahr 2016 gegründet wurde und seinen Hauptsitz in UK hat. Im Jahr 2018 machte das Unternehmen einen Umsatz von 7,8 Millionen Pfund und besaß 52 Mitarbeiter. Tenderlake bietet als „Smart Assistant" einen Service basierend auf künstlicher Intelligenz zur Analyse von Ausschreibungsbekanntmachungen in UK und Europa an.

Als Teil der Produktpalette bietet Tenderlake den European Procurement Data Atlas an, Tenderlake Foresight und Tenderlake Bespoke.

Der European Procurement Data Atlas ist eine webbasierte und interaktive Datenbank, die öffentliche Einkaufsdaten der letzten sechs Jahre einheitlich dargestellt in Euro beinhaltet (auf Anfrage sind auch Daten bis zu zehn Jahre rückwirkend erhältlich). Basierend hierauf können diverse Analysen zur Beantwortung zum Beispiel folgender Fragen bereitgestellt werden:

- Wie hoch ist die öffentliche Verausgabung in welchem Land und in welchen Industrien?

- Welcher Anteil der Verträge in Prozent wird außerhalb des Landes vergeben (pro Land und Industrie)?

- Nach welchem Muster werden Verträge (nach Anzahl und Volumen) in welchen Ländern und Industrien durch welche Art von Einkäufern vergeben? 
- Welche Produkte und Dienstleistungen lassen das meiste Wachstum in welchem Land und für welche Einkäufer erwarten?

- Wie ist die Verteilung auf welche Vertragsarten in welchen Ländern?

- Welcher Anteil der Angebote wurde über SMEs eingereicht - wie sieht die zu erwartende Entwicklung aus?

„Tenderlake Foresight“ analysiert historische Einkaufsmuster und bietet eine Aussage darüber, wer wahrscheinlich einen Bedarf an dem angebotenen Produkt/ Dienstleistung in den kommenden Monaten haben wird. Dies kann genutzt werden, um zum Beispiel Geschäftsentwicklungsmöglichkeiten stärker hierauf auszurichten und eine größere Anlagerendite zu erhalten.

„Tenderlake Bespoke“ beinhaltet die individuelle Beantwortung von Anfragen rund um die Nutzung öffentlicher Einkaufsdaten (Tenderlake Ltd., 2019d).

Tenderlake wird bereits von etwa 3500 Organisationen genutzt. Die Suche kann in 24 Sprachen erfolgen, während die Auftragsbekanntmachungen automatisch in Englisch übersetzt werden. Das Unternehmen war Gewinner des EU Datathons 2018 und wurde außerdem durch Google und die Financial Times als Top 100 „Digital Champion“ in Europa in der Kategorie „Using technology for social challenges" geehrt (Tenderlake Ltd., 2019a; The Financial Times Ltd., 2019). Mit Blick auf das Preismodell wird ein „Professional“-Paket und ein „Enterprise“-Paket angeboten (Tenderlake Ltd., 2019c).

\section{PublicBI - Echtzeitanalyse öffentlicher Einkaufsdaten}

Sitz des Unternehmens PublicBI bestehend aus zwei Personen ist Deutschland (PublicBI, 2018, S. 12). Es wurde 2017 gegründet und bietet Echtzeit-Analyse und Darstellung eines Dashboards über alle EU-relevanten öffentlichen Einkäufe an. Diverse Selektionsmöglichkeiten umfassen jene nach Land, Jahr, Verfahrensart, Lieferant und Vertragsart. Zielgruppen sind die Wirtschaft und Bürger (PublicBI, 2018, S. 15).

Die Daten basieren im Wesentlichen auf offenen Daten der EU der letzten zwölf Jahre. Diese wurden angereichert durch zum Beispiel Ländercodes, Sprachcodes, das BPI der Länder und Inflationsraten. Basierend auf einer Datenbereinigung können so Dashboards und Visualisierungen angeboten werden, die für alle zugänglich, interaktiv und cloudbasiert sind. Ihre Nutzung setzt keine Programmierkenntnisse voraus und die Öffentlichkeit kann Feedback und Anfragen im Sinne einer kollaborativen Entwicklung stellen (PublicBI, 2018, S. 8 f.). 
Das Angebot ist aktuell kostenfrei und soll in Richtung eines Geschäftsmodells weiterentwickelt werden. Ziel ist es, zum Beispiel vermehrt Trendanalysen anzubieten, mehrere Dimensionen abzubilden, mehrere Datensätze $\mathrm{zu}$ ergänzen und Korrelationen $\mathrm{zu}$ entdecken, die Datenqualität zu steigern, mit anderen Datenanbietern zusammenzuarbeiten und über Mustererkennung fehlende Werte zu ergänzen (PublicBI, 2018, S. 10). PublicBI war zweiter Gewinner des EU Datathons 2018.

\section{Schlussfolgerungen}

Die drei Beispiele zeigen eindrücklich die Anwendung neuer Technologien auf den öffentlichen Einkauf. Durch die bereits bestehende Anzahl der Nutzer, aber auch durch die Umsatzhöhe im Beispiel von Tenderlake nach nur drei Jahren, wird der Bedarf deutlich. Während C4P und Tenderlake schon etwas weiter fortgeschritten sind, steht PublicBI noch am Anfang seiner Entwicklungsmöglichkeiten. Aber auch dieses Unternehmen überzeugt bereits jetzt durch das frei verfügbare Angebot eines umfänglichen Echtzeit-Dashboards. Alle drei Beispiele könnten auch sehr gut für den deutschen Markt angewendet beziehungsweise ausgebaut werden.

\subsection{Zwischenfazit: Vielfalt bestehender Lösungsansätze auch für Deutschland nutzbar}

Die in diesem Kapitel, aber auch im Laufe der Arbeit vorgestellten Best Practices geben diverse Anregungen, wie die Öffnung des öffentlichen Einkaufs umgesetzt werden kann. Wichtig erscheint eine Einbettung in ein gesamthaftes Vorgehen bestehend aus mehreren Maßnahmen wie Frankreich und die Slowakei eindrücklich zeigen. Die vorgestellte Kriterienliste kann helfen, weitere Länderbeispiele zu bewerten und - bei weiterer Anwendung - Muster und Präferenzen zu erkennen. Einzelne Best Practices können ganzheitliche Maßnahmen vervollständigen und darüber hinaus gute Anregungen für eigene Geschäftsmodelle geben. 
Open Access Dieses Kapitel wird unter der Creative Commons Namensnennung 4.0 International Lizenz (http://creativecommons.org/licenses/by/4.0/deed.de) veröffentlicht, welche die Nutzung, Vervielfältigung, Bearbeitung, Verbreitung und Wiedergabe in jeglichem Medium und Format erlaubt, sofern Sie den/die ursprünglichen Autor(en) und die Quelle ordnungsgemäß nennen, einen Link zur Creative Commons Lizenz beifügen und angeben, ob Änderungen vorgenommen wurden.

Die in diesem Kapitel enthaltenen Bilder und sonstiges Drittmaterial unterliegen ebenfalls der genannten Creative Commons Lizenz, sofern sich aus der Abbildungslegende nichts anderes ergibt. Sofern das betreffende Material nicht unter der genannten Creative Commons Lizenz steht und die betreffende Handlung nicht nach gesetzlichen Vorschriften erlaubt ist, ist für die oben aufgeführten Weiterverwendungen des Materials die Einwilligung des jeweiligen Rechteinhabers einzuholen.

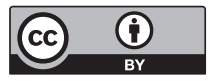

